\title{
Pratique basée sur la théorie des soins de fin de vie aux patients cancéreux: une exploration du modèle de soins infirmiers McGill
}

par David Kenneth Wright et Catherine Pugnaire Gros

\begin{abstract}
Abrégé
Les théories infirmières inspirent les praticiens en décrivant comment répondre aux besoins, rehausser le bien-être et interagir avec les clients en tant que personnes considérées dans leur globalité. Cet article examine le modèle de soins infirmiers McGill relativement à la façon dont ses principes peuvent appuyer la pratique infirmière à l'intention des patients cancéreux parvenus à la phase terminale. Nous avançons que ce modèle fournit une approche relationnelle tout à fait congruente avec la philosophie des soins palliatifs. Cet article vise à encourager la réflexion parmi les infirmières en oncologie et les dirigeantes infirmières. En comparant les valeurs fondamentales de notre pratique à la théorie des soins infirmiers existante, nous pourrions découvrir de nouvelles opportunités d'enseigner et d'apprendre en quoi consiste exactement la prestation de soins infirmiers de fin de vie.
\end{abstract}

«Si nous permettons à cette prochaine génération d'infirmières d'ignorer la pertinence de la théorie infirmière, une inquiétude qui a été récemment soulevée par divers observateurs, il se peut que nous fassions courir à notre profession le risque considérable de perdre son âme et sa vocation essentielle.»

(Thorne, 2007, p.348, traduction libre)

Chaque infirmière en oncologie est, ou devrait être, une infirmière en soins palliatifs. Malgré les progrès liés à l'innovation médicale et à l'allongement de la durée de survie, le cancer demeure une maladie mortelle dans bien des cas. Plus d'un quart de tous les Canadiens mourront du cancer, le nombre de décès par cancer étant estimé à 75000 en 2011 (Comité directeur sur les statistiques sur le cancer de la Société canadienne du cancer, 2011). Parmi les personnes touchées, certaines atteindront leur

\section{Au sujet des auteurs}

David Kenneth Wright, inf., M.Sc.(A), candidat au Ph.D., Unité de recherche et enseignement en soins infirmiers palliatifs, École des sciences infirmières, Université d'Ottawa, 451 rue Smyth, Ottawa, ON K1H 8M5.Dwrig079@uottawa.ca

Auteur à qui adresser toute correspondance : David Wright, 1514-296-7069. Adresse postale : 868 Sherbrooke E, Montréal, QC H $2 L 1 K 9$

Catherine Pugnaire Gros, inf., M.Sc.(A), Professeure adjointe, École de sciences infirmières, Université McGill, 3506 rue University, Montréal, QC H3A 2A7 fin de vie sans que leurs symptômes ne soient soulagés ni que leurs souhaits ne soient assouvis. Selon d'aucuns, les besoins palliatifs non satisfaits des patients en phase terminale constituent une crise au sein des soins de cancérologie, une crise que les infirmières en oncologie peuvent juguler du fait de leur position privilégiée (Coyne, Paice, Ferrell, Malloy, Virani et al., 2007).

La certification en soins infirmiers en oncologie exige des compétences fondamentales en soins de fin de vie. Il est attendu des infirmières en oncologie qu'elles comprennent, par exemple, les besoins particuliers des patients en fin de vie ainsi que les buts du traitement palliatif (Association des infirmières et infirmiers du Canada, 2009). Malheureusement, les infirmières n'ont pas toutes la formation requise pour soigner convenablement ces patients. Les environnements de santé dans lesquels nous prodiguons les soins aux patients atteints de cancer sont orientés vers la réussite du traitement et vers la guérison éventuelle de la maladie. Bien que cette approche soit importante et adéquate pour beaucoup de patients, elle tend également à détourner notre attention collective de la question de la mort. La méconnaissance du processus du mourir et le peu d'attention qui lui est porté peuvent entraîner un désengagement visà-vis de nos patients en fin de vie et une multitude d'occasions ratées.

Les soins palliatifs ne représentent pas un lieu mais une philosophie (Kessler, 2007, traduction libre). Ils constituent une approche qui promeut la qualité de vie, le confort et le mieuxêtre et réagit à la souffrance dans le contexte d'une maladie à issue mortelle. La philosophie des soins palliatifs est à la fois une affirmation de la vie et une reconnaissance de l'universalité et de la normalité de la mort. Elle milite en faveur du soutien à fournir aux proches des patients et de la considération des personnes dans leur globalité. Le soin global de la personne exige qu'on intègre attentivement les dimensions physiques, psychologiques, sociales et spirituelles de la santé, de la maladie et du mourir (Pastrana, Junger, Ostgathe, Elsner \& Radbruch, 2008).

Le concept des soins palliatifs n'est pas nouveau. Ce courant qui est né et s'est développé durant les années 1960 et 1970 à l'intérieur des soins de santé forme dorénavant une sous-spécialité médicale reconnue (Saunders, 2000). Les soins palliatifs se sont insérés dans le discours des praticiens en oncologie et l'on reconnaît de plus en plus ouvertement la nécessité d'incorporer les soins palliatifs aux systèmes de soins conventionnels (Clark, 2007). On attend donc de plus en plus des infirmières qu'elles dispensent des soins palliatifs dans les établissements de grande taille tels que des hôpitaux et les milieux de soins de longue durée. Ce sont d'ailleurs les contextes où meurent la majorité des Canadiens (Wilson, Truman, Thomas, Fainsinger, Kovacs-Burns et al., 2009). 
Le contexte actuel de la prestation des soins de fin de vie peut saper le sentiment d'identité professionnelle des infirmières et les amener à douter des fondements philosophiques d'une pratique infirmière optimale. Dans les milieux de soins qui ne sont pas explicitement axés sur le soin aux mourants, la responsabilité d'efficacité peut parfois l'emporter sur celle de prendre soin d'autrui. En outre, alors qu'une bonne partie du discours des soins palliatifs s'articule autour de récits convaincants sur la «bonne mort» mettant en jeu les thèmes de la paix, de la transcendance et de la "guérison», il nous faut faire attention de ne pas projeter de distorsion romantique sur le contexte réel de la prestation des soins en fin de vie. Dans leur pratique quotidienne, les infirmières qui soignent des mourants sont confrontées à une douleur, une tristesse et une souffrance incommensurables (Wright, Brajtman \& Bitzas, 2009). Elles œuvrent auprès de patients et de proches qui, souvent, souffrent et sont habités par la colère, la culpabilité et/ou la méfiance.

Les théories infirmières abordent les assises philosophiques de notre discipline. Elles proposent des idées explicites sur ce que sont les soins infirmiers et sur le rôle que jouent les infirmières; elles inspirent les praticiens en articulant comment répondre aux besoins, rehausser le bien-être et interagir avec les clients en tant que personnes considérées dans leur globalité (Fitzpatrick, 2005; Meleis, 2007). Les modèles théoriques en soins infirmiers constituent une sorte de prisme permettant d'interpréter et de mieux comprendre les situations qui surviennent dans la pratique et, aussi, d'analyser sa propre réaction à titre d'infirmière et d'y réfléchir. Cet article vise à examiner la congruence entre les idées tirées d'une approche théorique particulière des soins infirmiers, le modèle de soins infirmiers McGill, d'une part, et le contexte des soins de fin de vie, d'autre part. L'examen de cette congruence ouvre la voie à une réflexion sur les possibilités se présentant dans la pratique des soins infirmiers en oncologie destinés aux mourants.

\section{F. Moyra Allen et le modèle de soins infirmiers McGill}

La D ${ }^{\text {re }}$ F. Moyra Allen (1921-1996) était un éminent chef de file de la profession infirmière de grand renom au Canada et à l'étranger pour l'ensemble de ses travaux inspirés par la vision de «ce que les soins infirmiers peuvent être» (Stuart, 2002, p. 165, trad. libre). La $\mathrm{D}^{\mathrm{re}}$ Allen a consacré sa carrière à l'élaboration d'un cadre de référence pour les soins infirmiers qui les distingue du travail effectué par tous les autres types de prestataires de soins de santé (Gottlieb, 1995). Elle a d'abord intitulé ce cadre «modèle de développement de la santé» mais il est désormais connu sous le nom de modèle de soins infirmiers McGill (ou modèle McGill) (Gottlieb \& Rowat, 1987). La D ${ }^{\text {re }}$ Allen jugeait qu'à la suite de la réforme des soins de santé réalisée au Canada dans les années 1960 et du défi lancé à la profession infirmière d'assumer un rôle élargi, notre discipline était confrontée à un choix crucial. Nous pouvions diriger notre expansion vers le domaine de la médecine et nous charger de connaissances et de compétences du ressort traditionnel des médecins ou bien diriger notre expansion vers l'extérieur en développant un rôle complémentaire et unique en son genre qui se concentrerait sur le développement et le maintien de la santé de la famille (Allen, 1977). La $\mathrm{D}^{\text {re }}$ Allen souhaitait faire éclater le continuum linéaire santé-maladie sur lequel reposait le raisonnement biomédical conventionnel. Selon elle, la santé et la maladie doivent être perçues comme étant des variables distinctes: l'une ne correspond pas nécessairement à l'absence de l'autre. Comme elle l'a écrit, «la santé n'est pas une caractéristique statique, c'est une façon de vivre, d'être, c'est une façon de croître, de devenir» (Allen, 1981, p. 153, trad. libre). Pour elle, la définition de la santé comme étant l'absence de maladie présentait aux soins infirmiers leurs plus graves problème philosophique et dilemme logique. Si elle n'est que l'absence de maladie, la santé «n'a aucune signification propre ... On ne peut pas s'efforcer d'atteindre quelque chose qui n'existe que s'il $n$ 'est pas quelque chose d'autre» (Allen \& Warner, 2002, p.101, trad. libre, les mots en italique étaient mis en évidence dans la version originale).

Cette brève notice biographique illustre la pertinence des idées de la $\mathrm{D}^{\text {re }}$ Allen au domaine des soins infirmiers de fin de vie. Quoique la préoccupation centrale de ses écrits ne porte pas spécifiquement sur les soins de fin de vie, sa vision des soins infirmiers s'intéresse au soin et à l'accompagnement des patients et des familles à travers les transitions de la vie et les changements liés au développement. Tout comme la $\mathrm{D}^{\text {re }}$ Allen s'inquiétait de la médicalisation de la santé, la philosophie des soins palliatifs est née d'une insatisfaction vis-à-vis de la médicalisation du mourir (Clark, 2002). Ceci comprenait notamment les morts censément «violentes» que des individus éprouvaient en vertu d'un paradigme de la médecine de réanimation (Carnevale, 2005, p.2). La philosophie de l'approche palliative repose sur une notion de sauvetage, celle de sauver les patients mourants et leurs proches d'un système médical qui peut s'avérer froid, impersonnel et généralement à l'opposé de ce dont les individus ont vraiment besoin à l'approche de leurs derniers jours. On notera, à titre d'exemples, le besoin de ne pas éprouver de douleur physique, le besoin d'exprimer ses sentiments et ses émotions de façon individuelle, le besoin de recevoir des soins empreints de compassion, le besoin de faire une quête spirituelle et enfin, celui de mourir en paix et dans la dignité (Kessler, 2007). Selon la perspective partagée par la $\mathrm{D}^{\mathrm{re}}$ Allen et la philosophie des soins palliatifs, la maladie, la souffrance et la mort sont des situations humaines pouvant favoriser la survenue d'un apprentissage, d'une croissance et d'un développement (p. ex. la santé) personnels et familiaux. La santé est une caractéristique positive et elle peut exister au sein des expériences liées à la vie et à la mort, à la perte, au deuil et à la souffrance (Association canadienne de soins palliatifs, Comité des normes en soins infirmiers de l'ACSP, 2009).

\section{Le modèle de soins infirmiers McGill: concepts centraux}

Les concepts centraux du modèle McGill comprennent la santé, la famille, la collaboration et l'apprentissage. En bref, la santé est un construit multidimensionnel qui incorpore des processus de coping et de développement et qui tend vers le rehaussement de la qualité de vie et l'atteinte d'objectifs personnels et familiaux. La famille constitue le contexte d'apprentissage de la santé, et elle représente à ce titre un point de mire de l'évaluation et de l'intervention infirmières. Les soins infirmiers mettent en jeu un processus de collaboration au sein duquel les patients et leurs familles participent activement à la démarche de promotion de la santé (Gottlieb \& Rowat, 1987).

Le modèle McGill est essentiellement un modèle d'apprentissage puisque la promotion de la santé est considérée comme un processus continu d'interrogation et de découverte (PugnaireGros \& Young, 2007). Selon le modèle McGill, les soins infirmiers sont une science des interactions de promotion de la santé (Pugnaire, 1981) au sein desquelles les infirmières, les patients et leurs familles se rassemblent pour apprendre et travailler sur des situations de santé et de la vie quotidienne (Allen \& Warner, 2002). Le modèle McGill a été récemment modifié afin d'y intégrer le Developmental/Health Framework, une conceptualisation qui articule de façon plus exhaustive la nature de la personne et de l'environnement au sein du modèle théorique initialement 
proposé (Gottlieb \& Gottlieb, 2007). Animée par les philosophies liées aux soins holistiques, la pratique infirmière est, selon le Developmental/Health Framework et le modèle McGill, axée sur les forces. Cela exige que l'on reconnaisse, cultive et optimise les attitudes, compétences, ressources et aspects positifs inhérents à la personne/famille (Gottlieb \& Gottlieb, 2007). Les observations et les expériences cliniques sont interprétées au moyen d'un langage mettant en valeur les forces et le potentiel plutôt que de souligner les déficits ou les problèmes (Feeley \& Gottlieb, 2000).

\section{Le modèle de soins infirmiers McGill: Ontologie de la personne}

Le modèle McGill affirme que les personnes sont intrinsèquement libres de participer à leur propre expérience de santé et d'assumer la responsabilité des choix qu'elles font. En vertu du modèle McGill, les personnes sont des êtres pleinement conscients, flexibles et tournés vers la réflexion et la réciprocité qui sont en fin de compte «les architectes de leur propre expérience» (Gottlieb \& Gottlieb, 2007, p.E47, trad. libre). Ils sont intrinsèquement poussés vers la réglementation, l'attachement et le coping, parce que ces forces sont nécessaires à la survie (Gottlieb \& Gottlieb, 2007).

Le modèle McGill souligne le caractère indissociable de la personne et de son environnement, bien que l'environnement constitue un contexte discernable dans lequel les individus réalisent leur potentiel, reflétant par là-même une conception du développement humain nature via nurture, c'est-à-dire que l'inné joue un rôle avec l'aide de l'environnement. Les soins infirmiers visent à promouvoir le meilleur degré d'ajustement entre les individus et leurs environnements, et donc à faciliter une adaptation réussie (Gottlieb \& Gottlieb, 2007, p.E48).

Dans le modèle McGill, il est entendu que la personne se situe immanquablement dans le contexte familial et, dans cette perspective, c'est la famille qui constitue «l'unité d'intérêt » (Gottlieb \& Rowat, 1987, p. 55). Même lorsque l'infirmière interagit avec une seule personne (p. ex. le patient), elle perçoit cette personne à travers le prisme relationnel de la famille, en reconnaissant l'influence réciproque de l'individu et de sa famille. L'individu et la famille sont considérés comme «des systèmes ouverts en constante interaction entre eux et avec d'autres systèmes au sein de leur environnement» (Gottlieb \& Rowat, 1987, p.55, trad. libre). Le principe théorique des vies liées expose la nature fondamentalement relationnelle de l'expérience et du développement humains, soulignant par là même que les individus existent au sein d'une toile de relations significatives. Le modèle théorique précise que:

"Les choix d'un individu sont souvent influencés par ce qui se passait ou se passe dans la vie d'autres personnes. Chaque individu suit sa propre trajectoire de développement, et cette trajectoire est solidaire de celles d'autres membres de ses réseaux sociaux. Lorsque les soins infirmiers embrassent ce principe, l'infirmière élargit et approfondit le point de mire des soins en s'occupant de multiples priorités et voyant le tout à travers un prisme intergénérationnel» (Gottlieb \& Gottlieb, 2007, p.E49, trad. libre).

\section{Le modèle de soins infirmiers McGill: ses rapports avec d'autres théories et modèles infirmiers}

Le modèle McGill a des idées en commun avec d'autres théories des soins infirmiers. Ainsi, la plupart des théories infirmières et des modèles théoriques en soins infirmiers partagent des valeurs communes telles que l'holisme, l'humanisation, le sens, la qualité de vie, la santé et le confort (Meleis, 2007; Willis, Grace \& Roy, 2008). Cependant, les perspectives théoriques divergent en fonction de l'accent mis sur des valeurs et concepts particuliers et de l'articulation de l'objectif primaire et de la raison d'être des soins infirmiers. Meleis (2007) cerne quatre écoles de pensée qui fournissent un cadre d'orientation pour la panoplie de théories infirmières existantes. Selon son analyse, le premier groupe de théoriciens appartient à l'école des besoins (p. ex. Henderson, Orem). Ces théoriciens se concentraient sur les problèmes, les déficits et les besoins non satisfaits des clients des soins infirmiers. Le deuxième groupe de théoriciens est lié à l'école de l'interaction (p. ex. Paterson et Zderad, Peplau). Ces théoriciens ont complété les questions soulevées par l'école des besoins et ont porté leur attention théorique sur la relation entre le client et l'infirmière. Ils posaient les soins infirmiers comme étant un processus interpersonnel survenant entre une personne ayant besoin d'aide et une personne capable de lui fournir cette aide. Le troisième groupe concerne l'école des effets souhaités (p. ex. Johnson, Rogers, Roy). Ses théoriciens s'intéressaient prioritairement au pourquoi des soins infirmiers, aux résultats finals pouvant être atteints par le truchement de la démarche infirmière. Ils s'attachaient à la relation du client à son environnement et assignaient aux soins infirmiers un rôle de fourniture d'équilibre, de stabilité et d'adaptation. Enfin, les théoriciens de l'école du caring (Watson et Parse) ont introduit la notion selon laquelle les soins infirmiers sont une activité relationnelle axée sur les rapports, le dialogue et la présence.

D'après l'organisation de Meleis, dans les trois premières écoles de pensée ci-dessus, la prise de décision échoit principalement au prestataire de soins. À cet égard, le modèle McGill rejoint le seul paradigme du caring en ce qu'il accorde une priorité primaire et fondamentale aux clients en tant qu'architectes de leur propre expérience et que responsables ultimes des décisions qui les touchent. En revanche, le modèle McGill s'éloigne de la doctrine de l'école du caring dans son approche du coping et du développement à titre de concepts théoriques centraux. Parse, par exemple, conçoit la santé comme un engagement personnel que chaque être humain vit en incarnant ses priorités de valeur dans son rôle d'auteur (Parse, 1990). Elle écrit ainsi que la santé «n'est pas une entité linéaire qui peut être interrompue ou qualifiée à l'aide de termes tels que bonne, mauvaise, plus ou moins. Elle ne consiste pas à s'adapter à l'environnement» (Parse 1998, p.32, trad. libre, les mots en italique étaient mis en évidence dans la version originale). Selon le modèle McGill, les individus progressent vers une meilleure santé par le biais des processus de coping et de développement. Le coping est la manière dont les individus réagissent aux situations et le développement se rapporte à l'atteinte des buts dans la vie (Gottlieb \& Rowat, 1987).

Examiné à la lumière des autres théories infirmières existantes, le modèle McGill se distingue par l'utilisation qu'il fait d'un cadre d'apprentissage pour les soins infirmiers et pour la promotion de la santé et par la prépondérance de concepts particuliers et leur organisation. Le modèle McGill préconise une philosophie de la pratique qui est axée à la fois sur le patient et sur la famille, sensible à la situation et fondée sur la collaboration. La prépondérance accordée aux soins axés sur la famille est cohérente avec les philosophies formulées des soins infirmiers familiaux (p. ex. Wright \& Leahey, 2004). Le modèle McGill situe l'infirmière en tant qu'apprenante, partenaire et accompagnatrice tandis que les clients traversent les épreuves de santé et de maladie. Le rôle de l'infirmière consiste à aider les individus à progresser vers un bien-être et une qualité de vie rehaussés en fonction de modalités qu'ils définissent eux-mêmes. 


\section{Exemple tiré de la pratique clinique}

"...un jour, j'ai œuvré auprès d'une jeune femme atteinte d'un cancer très avancé. Je n'ai jamais vu de ma vie une personne tellement décharnée. Mais il y avait quelque chose à propos de cette femme-un état d'esprit sans pareil. J'ai pris soin d'elle durant la matinée et vers midi, je lui ai dit spontanément, "vous savez, vous avez un esprit extraordinaire». Tout en disant cela, je pensais également: "Quelle ironie qu'une telle chaleur et qu'un tel esprit émanent d'un corps aussi malmené». Elle s'est alors rendue, bien difficilement, jusqu'à son bureau et en a tiré une enveloppe fermée. Elle m'a montrée une photo d'elle qui avait été prise dix mois auparavant alors qu'elle était en vacances. On y voyait une fermme souriante en maillot de bain qui révélait ses courbes voluptueuses. C'était sa façon de me dire «Voici qui je suis». La photo avait saisi la force d'esprit que j'avais moimême observée. Ce fut le point de départ de notre conversation sur les multiples changements qu'elle avait éprouvés. Le plus grand d'entre eux était l'incroyable modification de son apparence physique. J'ai vite appris de notre discussion que son but était de ne pas céder à l'amertume. Elle a ainsi déclaré: "Je ne veux pas devenir amère au sujet de tout cela, je veux demeurer qui je suis, conserver cet état d'esprit, l'essence de mon être malgré ce que les gens voient à l'extérieur. "Je venais à peine de rencontrer cette femme quand cet échange s'est produit, mais parce que j'avais appris à la connaître rapidement, ainsi que ses buts, notre relation de collaboration s'est poursuivie pendant quelques semaines.» (Gottlieb \& Feeley, 2005, p. 114; passage dû à Heather Hart, une infirmière en soins palliatifs, trad. libre).

Dans cet exemple, l'infirmière est prête à être émerveillée par ses patients. Elle cerne la force d'esprit d'une femme chez qui d'aucuns ne verraient que maladie, faiblesse et mourir. En faisant l'éloge de cette force, l'infirmière déblaie un espace où pourront survenir apprentissage et exploration. La patiente choisit de partager sa photographie et de participer à un dialogue où elle approfondit les changements qu'elle a subis. Grâce à cette démarche, l'infirmière parvient à comprendre sa patiente, à bien saisir ses priorités et à découvrir son but en matière de santé.

Cet exemple est éloquent parce qu'il illustre comment une démarche infirmière qui vise à cultiver les forces et à favoriser la collaboration amène la prestataire à connaître ses clients à un niveau radicalement différent de ce qui est autrement possible. Comprendre le but que s'est donné cette mourante peut alors orienter la planification et la prestation de soins véritablement axés sur le patient.

\section{Intégration du modèle McGill aux soins en fin de vie}

Dans les sections qui suivent, deux dimensions de la philosophie de la pratique tirées du modèle McGill sont examinées en lien direct avec le contexte des soins en fin de vie : 1) transitions et niveau de préparation, et, 2) soins axés sur la famille.

\section{Transitions et niveau de préparation}

Quoiqu'ils s'appliquent aux soins infirmiers en général, les concepts de transitions développementales et situationnelles revêtent une importance particulière dans les contextes de fin de vie. Selon Twycross (2007), l'objectif fondamental des soins de fin de vie est d'aider les patients et les familles à faire la transition «de la lutte contre la mort à la recherche de la paix» (p.9, trad. libre). Pourtant, les limites entre les approches purement «curatives» (thérapie visant à agir sur la maladie dans le but de prolonger la vie) et les approches purement «palliatives » (thérapie visant à agir sur les symptômes dans le but de rehausser la qualité de vie) ne sont jamais nettes ni absolues. Par exemple, beaucoup de patients en oncologie reçoivent des traitements anticancéreux conçus pour rehausser la qualité de vie voire prolonger la survie, même s'ils ne sont pas faits pour guérir la maladie. Les infirmières jouent un rôle central en aidant les patients et leurs proches à s'y retrouver dans le changement d'objectif des soins à la fois délicat et graduel accompagnant l'évolution de la maladie et l'approche de la mort.

D'après une étude basée sur l'analyse par théorisation ancrée qui explorait les déterminants de soins optimaux en fin de vie du point de vue d'infirmières d'hôpital, la «création d'un havre pour un passage sans heurts» est un thème fondamental des soins optimaux en fin de vie (Thompson, McClement \& Daeninck, 2006, p.93, trad. libre). Plusieurs obstacles à la réussite de la transition («le changement de file» des soins curatifs aux soins palliatifs) ont été dégagés dans cette étude. Il s'agissait notamment des suivants: 1) le déni de la mort par les infirmières, les médecins, les patients et leurs familles, 2) la difficulté d'établir des pronostics exacts, et enfin, 3) les perspectives divergentes sur ce qu'on entend par soins optimaux de fin de vie dans les contextes de soins. Inversement, la réussite de la transition est facilitée lorsque les patients, leurs familles et les prestataires de soins ont tous conscience de l'imminence de la mort et qu'ils l'acceptent pleinement:

«... les infirmières, les médecins, les patients et leurs proches doivent clairement comprendre que la personne touchée ne se rétablira pas de sa maladie et que le pronostic est extrêmement sombre ... quand les patients et leurs proches comprennent clairement la nature et le pronostic de la maladie et quand les discussions sur la fin de vie se produisent alors que le patient est encore capable de participer à la prise de décision, les infirmières indiquaient que le changement de file se passait plus rapidement et plus en douceur» (p.95, trad. libre).

Les infirmières ayant participé à cette étude soulignent le rôle important que les soins infirmiers peuvent jouer dans l'accompagnement des patients et des familles dans leur cheminement de fin de vie, notamment en allégeant le fardeau de la perte imminente par la fourniture d'information et de soutien. Par contre, elles sont plus silencieuses sur la meilleure façon d'aider les patients et leurs familles qui ne sont pas prêts, ne désirent pas-ou-refusent de reconnaître l'imminence de la mort. Pour certains d'entre eux, discuter avec les professionnels de la santé de l'acceptation de leur propre mourir ou du mourir d'un membre de la famille peut ne pas correspondre à leurs besoins. Il arrive peut-être trop fréquemment que les soins infirmiers imposent de formidables attentes aux patients en phase terminale et à leurs familles en exigeant qu'ils lâchent prise face à l'inévitabilité du mourir (Lowey, 2008). D'un autre côté, nous semblons être moins aptes à manifester tolérance et respect vis-à-vis des modes d'adaptation divergents que sont l'espoir, l'évitement ou le déni. Ce que nous nommons problématique peut en fait être ce qui convient le mieux à certains patients et à certaines familles. Une récente étude réalisée par Sjolander, Hedberg, Ahlstrom et al. (2011) rapporte que, dans le contexte d'un diagnostic de cancer avancé, certaines familles font front commun dans le but de chasser toute pensée de perte imminente. Ces familles «ne savent pas combien de temps la personne a encore à vivre, et elles ne veulent pas vraiment le savoir non plus» (p.3-4, trad. libre, le mot en italique était mis en évidence dans la version originale). En effet, une bonne mort est grandement individuelle et variable-pour une personne donnée, une bonne mort peut signifier renoncement, acceptation et paix tandis que pour une 
autre, cela voudra dire pouvoir revendiquer s'être accrochée à la vie jusqu'à son dernier soupir. Comme Quill, Arnold et Back (2009) l'ont observé,

"Il y a des patients et des familles qui accordent une bien plus grande valeur au prolongement de la vie qu'à l'évitement des souffrances inutiles; il y en a d'autres qui ne font pas suffisamment confiance au système médical pour abandonner un quelconque traitement, et d'autres encore qui ne souhaitent pas être confrontés à l'éventualité de mourir. Une fois que le patient articule clairement sa philosophie en faveur de tous les traitements possiblement efficaces, même s'ils sont pénibles ou invasifs, tout poursuite des négociations visant à fixer des limites est peu susceptible de produire des résultats et peut paraître abusive» (p.348, trad. libre).

La pratique infirmière imprégnée du modèle McGill s'inscrit explicitement au sein d'un cadre de pratique sensible à la situation en vertu duquel les interventions sont personnalisées afin de les rendre congruentes au niveau de préparation du client et de les dispenser aux moments optimaux (Dalton \& Gottlieb, 2003; Gottlieb \& Feeley, 2005). Lorsque les individus et les familles envisagent le changement, l'infirmière et le client participent à une démarche de réflexion en collaboration. Les interventions comprennent le recadrage d'enjeux qui peuvent être considérés sous un jour nouveau, et la sensibilisation en mettant en évidence les similitudes et les différences entre diverses situations et en soulignant les changements observés (Gottlieb, 1997). Lorsque les familles franchissent la transition vers la fin de vie et qu'elles se voient offrir les soins palliatifs de «réconfort» comme option thérapeutique, l'infirmière pourrait, par exemple, recadrer la croyance selon laquelle les soins palliatifs signifient «qu'on ne peut plus rien faire» en la remplaçant par la perspective qui veut que les soins palliatifs soient synonymes de gestion agressive des symptômes et d'accompagnement rapproché et régulier à la fois pour le patient et pour sa famille.

L'infirmière dont la pratique s'inspire du modèle McGill comprend bien que les patients et les familles peuvent être prêts à changer certains aspects de leur situation mais pas d'autres; le niveau de préparation est à la fois un état et une démarche. Cette réalisation est cruciale pour la prestation efficace de soins infirmiers de fin de vie étant donné que les soins palliatifs ne constituent pas une approche tout ou rien. Ils représentent plutôt un paysage complexe d'activités de la vie quotidienne où les gens continuent de prendre des décisions relativement à divers aspects de leurs soins et de leur vie. Il existe en effet une grande variation parmi les familles et les individus en ce qui concerne la mesure dans laquelle ils «se battent contre la mort» ou «recherchent la paix» à l'approche de la mort. Nous autres infirmières devrions continuellement nous demander où en sont le patient et ses proches et ce que nous pouvons faire, ensemble, pour offrir le maximum d'aide et de soutien à ce stade particulier. Les patients et les familles vivant des situations de fin de vie jouissent d'une pleine habilitation, maintiennent ou retrouvent un sentiment de contrôle grâce aux interventions infirmières qui leur donnent des occasions de faire le deuil de leurs pertes, de faire face à un avenir incertain et de célébrer les réussites et réalisations de toute une vie.

Lorsqu'elles assimilent le concept de la préparation présenté dans le modèle McGill, les infirmières sont appelées «à évaluer le niveau de préparation afin de préparer leurs clients à travailler sur leur santé, ... à créer les conditions favorisant le niveau de préparation et ... à assumer les rôles qui aident les patients à se préparer au changement» (Dalton \& Gottlieb, 2003, p.115 (trad. libre). Le modèle McGill préconise aux infirmières œuvrant auprès de patients en fin de vie et de leurs familles de suivre l'exemple de leur client concernant la mesure dans laquelle le travail sur l'acceptation de la mort imminente-au moyen de conversations, de la résolution de problèmes et de la prise de décision-est réellement désirable et utile. Plutôt que d'encourager les patients et leurs proches à reconnaître leur situation et à y faire face, l'infirmière concentre son attention sur sa propre attitude, en acceptant le cheminement de son client et en reflétant son mode d'adaptation. Cette approche est illustrée par le compte rendu clinique rédigé par une infirmière en oncologie prodiguant des soins à un jeune homme du nord de l'Alberta:

"Il était plutôt avare en mots. Ce n'était pas son style. Je savais que dialoguer serait trop pour lui. Ce n'était pas nécessaire. Quand j'observais ses proches autour de lui, surtout sa mère qu'il aimait beaucoup, ils étaient tous très taciturnes. Ils se contentaient de rester assis auprès de lui. Ce qui fait que quand je lui prodiguais des soins, j'essayais de modeler mon comportement sur le leur... [afin de] manifester du respect pour la position qu'il adoptait, pour sa culture, sa manière d'être...» (Perry, 1998, p. 22, trad. libre).

Dans cet exemple, l'objectif de soin revient à faire confiance au patient et à sa famille à titre d'experts sur tout ce qui les concerne et à honorer qui ils sont et ce qu'ils font à ce moment précis. L'infirmière se met à leur disposition et leur fournit un accompagnement. Elle offre son expertise lorsqu'il convient et n'impose pas de programme inopportun. Du point de vue de l'infirmière, l'acceptation signifie découvrir et épouser les caractéristiques propres à chaque client/famille, offrir présence et intervention en utilisant une approche attentiste (Feeley \& Gottlieb, 1998).

\section{Soins collaboratifs axés sur la famille}

Une perspective où les soins sont axés sur la famille constitue le fondement des soins infirmiers en fin de vie (Chekryn Reimer, Davies \& Martens, 1991). L'appui fourni aux familles durant les phases terminales de la maladie des patients a une incidence positive sur l'ajustement au deuil (Dumont, Dumont \& Mongeau, 2008). Selon la constatation de Fanslow-Brunjes (2008),

"le mourant et sa famille et autres êtres chers [doivent] être considérés comme une seule unité, tout comme on le fait souvent avec la mère et son enfant. Il convient de les percevoir de cette façon car, d'une façon particulière et originale, ils se meurent tous» (p.81, trad. libre).

En outre, notre orientation vers la perspective et les besoins de la famille est une marque de respect à l'égard du patient luimême. En honorant les relations et les êtres qui lui sont chers, nous reconnaissons voir dans notre client un être relationnel pour qui d'autres individus ont revêtu de l'importance et qui revêt encore de l'importance pour autrui.**

Quoiqu'il soit généralement entendu que les soins axés sur la famille font partie intégrante de la philosophie des soins palliatifs, ils représentent souvent davantage un idéal qu'une réalité dans la pratique (Kristjanson \& Aoun, 2004). Les modèles théoriques en soins infirmiers qui soulignent l'importance de la famille sont donc d'une grande valeur pour la pratique des soins

‘Dans le cas de certains patients, il se peut qu'aucun membre de la famille ne soit présent. Pour d'autres, il est possible qu'il n'y ait aucune famille. Cette discussion sur les « soins infirmiers axés sur la famille » se réfère à la prestation de soins aux individus 
de fin de vie en ce qu'ils approfondissent notre compréhension du patient individuel puisque ce dernier se situe invariablement dans un contexte familial original. De plus, les approches axées sur la famille permettent aux infirmières d'intervenir directement auprès des membres de la famille qui ont leurs propres besoins de soutien dans ces situations de fin de vie.

Bien que la majorité des conceptualisations des soins infirmiers abordent les besoins de la famille d'une manière ou d'une autre, le modèle McGill inclut les relations familiales au sein même de sa conceptualisation de la personne (rappelant en cela le concept des vies liées). Selon Gottlieb et Gottlieb (2007),

"[Moyra] Allen avançait que l'unité de soins est la famille plutôt que le seul individu puisque c'est au sein de la famille que les individus font leur apprentissage sur la santé (c.-à-d. comment s'adapter) et que chacun des problèmes est un phénomène familial. Dans notre nouvelle conceptualisation, les soins infirmiers tournent leur attention à la fois vers l'individu et vers sa famille; l'individu considéré dans le contexte de la famille ainsi que la famille considérée comme unité de soins. Membre de la famille est défini dans le modèle McGill comme étant toute personne que le patient désigne comme faisant partie de sa famille. Quoique l'infirmière n'œuvre pas toujours auprès de la famille en tant qu'unité de soins, l'infirmière garde la famille à l'esprit et bon nombre des interactions infirmière-patient sont à tout le moins examinées dans une optique familiale, le cas échéant» (p.E54-55, trad. libre).

Le modèle McGill décrit explicitement le partenariat de collaboration établi avec les familles en tant que composante essentielle de la relation infirmière (Gottlieb \& Rowat, 1987). Un partenariat de collaboration amène l'infirmière et la famille à œuvrer ensemble à l'atteinte de buts définis en toute mutualité, par le truchement de démarches de négociation et de partage du pouvoir (Gallant, Beaulieu \& Carnevale, 2002). Selon l'approche de soins prônée dans le modèle McGill, les familles possèdent en elles-mêmes des forces et des ressources qui lui donnent la capacité de faire face aux défis de santé (Feeley \& Gottlieb, 2000). Les stratégies infirmières visant à promouvoir une pratique en collaboration axée sur la famille et sur ses forces dans l'optique du modèle McGill, comprennent notamment solliciter activement la perspective et les croyances de la famille et aider les membres de la famille à explorer, clarifier leurs besoins et leurs valeurs. Cette démarche de soins exige une ouverture d'esprit et le respect de la famille ainsi qu'une attitude d'acceptation, de la souplesse et une absence totale de jugements négatifs (Gottlieb \& Feeley, 2005).

La collaboration avec les patients et leurs proches constitue la pierre angulaire d'une pratique efficace des soins infirmiers de fin de vie. Le patient n'est pas seul à avoir besoin de soin : il est essentiel que les membres de la famille reçoivent eux aussi attention et soins durant cette période synonyme de transition et de changement pour eux (Chekryn Reimer, Davies \& Martens, 1991). En outre, les infirmières en oncologie ouvrent souvent auprès de familles de patients qui ne peuvent plus exprimer leurs souhaits (p. ex. patients comateux, patients sous sédation ou pour lesquels la mort est réellement imminente). Dans ces situations-là, les familles deviennent un atout important pour l'infirmière qui fait la connaissance du patient par l'intermédiaire de sa famille (p. ex. sa personnalité, sa biographie, ses préférences, ses valeurs).

Intervenir auprès de la famille signifie soutenir la participation des proches aux soins de leur être cher ainsi que leur participation à la prise de décision. À la fin de la vie, des choix sont faits de manière continuelle, et les familles font invariablement partie intégrante du processus décisionnel. De plus, une fois le patient décédé, ce sont les proches qui doivent vivre avec les décisions qui ont été prises. Il s'agit notamment des décisions concernant la gestion de la douleur, de la manière de réagir aux diverses circonstances à mesure qu'elles surviennent (p. ex. infection, déshydratation/malnutrition, trouble métabolique) et s'il convient ou non d'aborder avec le patient l'imminence de sa mort (Callanan, 2008). D'autres décisions moins spectaculaires sont prises constamment à mesure que s'organisent les soins infirmiers destinés au mourant et les familles apprécient généralement pouvoir faire entendre leur voix concernant ces décisions. Par exemple, l'infirmière devrait-elle retourner et repositionner le patient maintenant ou bien revenir le faire un peu plus tard? Serait-il préférable qu'elle aide à baigner le patient dans la matinée ou dans la soirée?, et ainsi de suite.

Les membres de la famille ne forment pas un ensemble homogène mais plutôt un groupe varié d'individus ayant des voix, perspectives et intérêts multiples (Meiers \& Brauer, 2008). Les infirmières prodiguant des soins aux familles dont un membre est en phase terminale œuvrent ainsi dans un paysage aux multiples vérités et réalités; il n’y tout simplement pas une seule bonne façon de considérer un problème ou une situation donné(e) (Leahey \& Harper-Jaques, 1996). La perspective tirée du modèle McGill appuie utilement les soins infirmiers dans ce contexte puisqu'elle accorde la priorité au développement de partenariats de prestation de soins avec les membres de la famille. Ces relations permettent d'encourager l'expression de perspectives possiblement divergentes et de partager et d'explorer ces dernières. Cela signifie apprendre auprès des familles dans le cadre d'interventions comme écouter et poser des questions pertinentes plutôt que jouer le rôle d'experte et dire aux proches ce qu'il faut faire (Gros \& Ezer, 1997). L'infirmière se donne comme priorité de faire confiance à la famille au lieu de tenter d'amener la famille à lui faire confiance (Pugnaire-Gros \& Young, 2007). Ainsi, en s'impliquant ensemble dans une démarche de découverte et d'apprentissage, l'infirmière dont la pratique s'inspire de la perspective prônée par le modèle McGill, œuvre auprès des membres de la famille d'une manière qui reflète l'approche qu'elle utilise avec le patient individuel (Gros \& Ezer, 1997).

Travailler auprès des familles faisant face à des situations de fin de vie n'est ni facile ni simple. Il se peut que les infirmières aient de la difficulté à accepter ou même à comprendre les attitudes, comportements ou approches des membres de la famille (Wright et al., 2009). Il est possible que les proches s'ingèrent dans les soins que l'infirmière s'efforce de prodiguer (p. ex. interdire l'administration d'un médicament ayant pour but d'assurer le confort du patient). Ceci peut grandement frustrer l'infirmière qui pourra réagir en adoptant une attitude relationnelle de confrontation ou d'évitement. Mais comme Cicely Saunders nous le rappelle, la façon dont les gens meurent reste gravée dans la mémoire de ceux qui leur survivent. Les soins infirmiers que nous dispensons lors des derniers mois, jours, heures et minutes de la vie d'un patient sont appelés à faire partie des récits qui seront racontés à maintes reprises par les proches tandis qu'ils se créent et vivent leur deuil. Les infirmières en oncologie ont le devoir éthique de personnaliser leurs soins de manière à ce qu'ils apportent aide et soutien aussi bien aux proches qu'au patient individuel. À cet égard, il est utile de disposer d'un cadre théorique plaçant l'expérience morale de la famille au centre des phénomènes d'importance pour la pratique infirmière. Comme la citation ci-dessous l'indique, l'étape du mourir peut produire des scènes horribles dont les gens se souviendront. Le fait de rester avec les patients et d'être témoins de leur souffrance est essentiel à la création d'un contexte pour l'expérience du mourir vécue par le patient et par sa famille.

"Elle était tellement malade. Elle se vidait de son sang devant mes yeux et il n'y avait pas grand-chose que je puisse faire, ni quiconque d'autre, d'ailleurs. Alors que je l'aidais à se 
remettre au lit, ses trois beaux fils adolescents se sont reculés vers le mur et l'ont regardée, horrifiés. ... Je me suis assise sur son lit et j'ai pris sa main dans la mienne en la serrant fort. En écartant de mon cerveau toute pensée... j'ai laissé mon énergie s'écouler vers elle. À mesure que les secondes s'égrenaient en silence, j'ai senti venir en moi une partie de son âme. Ce fut un moment de transformation pour moi, C'est à ce moment précis que je suis devenue infirmière» (Perry, 1998, p.11, trad. libre).

\section{Discussion}

Le modèle McGill inspire les infirmières œuvrant auprès des patients en phase terminale du cancer et leurs familles à adopter une approche relationnelle en harmonie avec la philosophie des soins palliatifs. La position prise par l'infirmière en est une de réconfort, de soutien et de présence régulière qui, loin d'imposer un programme particulier, offre accompagnement, respect et compréhension. Cette relation devient le contexte favorisant l'apprentissage et la découverte mutuels. L'infirmière est bienveillante, ouverte à l'autre, souple, curieuse et engagée. Elle incarne les valeurs fondamentales du modèle McGill et voit à leur réalisation: collaboration, pratique axée sur les forces, apprendre avec les patients et leurs proches tandis qu'ils traversent les situations quotidiennes associées à la fin de vie.

Les idées tirées du modèle McGill nourrissent discussions et débats parmi les infirmières en oncologie sur les significations des soins infirmiers axées sur la collaboration et sur la famille, sur le travail effectué auprès des patients et de leurs proches vivant des transitions et, finalement, sur la manière dont nous pouvons le mieux servir les communautés dans lesquelles nous exerçons. La nature collaborative des soins infirmiers et leur sensibilité aux situations telles que décrites dans le modèle McGill et telles qu'appliquées à la pratique infirmière en phase terminale, pourraient inspirer en nous un sentiment d'humilité autour de ce qui constitue une bonne mort et de notre rôle de facilitation en la matière. Il est possible que nous soyons fortement attachées à nos propres idées sur ce que bien mourir veut dire. Nous pouvons être persuadées que notre expérience nous donne le droit d'encadrer les patients et leurs familles vers leurs propres expériences en matière de bonne mort. Néanmoins, il nous faut être conscientes du rôle que nous jouons dans les expériences du mourir des autres. En dernier ressort, notre rôle consiste à exprimer aux personnes mourantes qui nous sont confiées et

\section{RÉFÉRENCES}

Allen, M. (1977). Comparative theories of the expanded role in nursing and implications for nursing practice: A working paper. Nursing Papers, 9, 38-45.

Allen, M. (1981). The health dimension in nursing practice: Notes on nursing in primary health care. Journal of Advanced Nursing, 6, 153-154.

Allen, F.M., \& Warner, M. (2002). A developmental model of health and nursing. Journal of Family Nursing, 8(2), 96-135.

Association des infirmières et infirmiers du Canada. (2009). Certification infirmière en oncologie : Plan directeur de l'examen et compétences de la spécialité. Récupéré à http://www2.cna-aiic. ca/CNA/documents/pdf/publications/CERT_Oncology_2009_ f.pdf

Callanan, M. (2008). Final journeys. A practical guide for bringing care and comfort at the end of life. New-York,NY: Bantam.

Carnevale, F.A. (2005). The palliation of dying: a Heideggerian analysis of the "technologization" of death. Indo-Pacific Journal of Phenomenology, 5(1), 1-12. à leurs proches qu'elles comptent pour nous, et que nous nous engageons à les aider à vivre leur expérience de fin de vie de la manière qu'ils l'entendent, selon leurs propres termes. Ces idées sont illustrées dans la réflexion suivante d'une infirmière en oncologie:

«Faire sentir au patient qu'il est la personne la plus importante au monde, même si ce n'est que durant les moments que vous passez auprès de lui, voilà ce qui devrait être notre but... j'en ai fait mon but... Ce sont les petites choses qui aident le patient à se sentir important, tel que la façon dont vous pénétrez dans la pièce. Je ralentis consciemment mes pas lorsque j'entre. Je prends le temps de m'asseoir dans la chambre du patient et d'écouter réellement ses préoccupations...Vous lui laissez savoir que sa vie compte encore. Même si ce n'est que pour cet instant, il compte pour quelqu'un» (Perry, 1998, p. 119, trad. libre).

\section{Conclusion}

La prestation de soins holistiques et intégrés aux patients et aux proches qui traversent le continuum des soins de cancérologie exige une collaboration entre la sphère oncologie et la sphère soins palliatifs de la pratique infirmière. Les infirmières en oncologie et les infirmières en soins palliatifs ont beaucoup à apprendre les unes des autres et à s'enseigner mutuellement tandis qu'elles s'efforcent de répondre aux besoins complexes de leurs patients atteints de cancers avancés. La philosophie des soins palliatifs, qui constitue essentiellement un ensemble d'idées concernant la reconnaissance de la mort et le maintien de la qualité de vie en dispensant des soins holistiques et axés sur la personne/famille, peut être adoptée avantageusement par toutes les infirmières œuvrant dans les milieux oncologiques. En utilisant le modèle McGill comme cadre de référence, les infirmières en oncologie peuvent découvrir de nouvelles façons d'exercer qui les amènent à demeurer fidèles à la philosophie des soins palliatifs sous-jacente, à la garder en tête puisque leur objectif final est d'apporter la plus grande assistance possible aux personnes faisant face à des situations de fin de vie.

\section{Remerciement}

Nous souhaitons remercier Andrea Witkowski, inf., M.Sc.(A), CSIO(C), de sa rétroaction et de ses commentaires mûrement réfléchis concernant une ébauche de cet article.

Chekryn Reimer, J., Davies, B., \& Martens, N. (1991). The nurse's role in helping families through the transition of "fading away". Cancer Nursing, 14(6), 321-327.

Clark, D. (2007). From margins to centre: a review of the history of palliative care in cancer. Lancet Oncology, 8, 430-438.

Clark, D. (2002). Between hope and acceptance: the medicalisation of dying. British Medical Journal, 324, 905-907.

Comité des normes en soins infirmiers de l'Association canadienne de soins palliatifs. (2009). Normes de pratique canadiennes en soins infirmiers palliatifs, 2009. Récupéré à http://acsp.net/ media/34575/Normes_de_pratique_Canadiennes_en_soins_ infirmiers_palliatifs_2009.pdf

Coyne, P., Paice, J.A., Ferrell, B.R., Malloy, P., Virani, R., \& Fennimore, L.A. (2007). Oncology end-of-life nursing education consortium training program: Improving palliative care in cancer. Oncology Nursing Forum, 34(4), 801-807.

Dalton, C.C., \& Gottlieb, L.N. (2003). The concept of readiness to change. Journal of Advanced Nursing, 42(2), 108-117. 
Dumont, I., Dumont, S., \& Mongeau, S. (2008). End-of-life care and the grieving process: family caregivers who have experienced the loss of a terminal-phase cancer patient. Qualitative Health Research, 18(8), 1049-1061.

Fanslow-Brunjes, C. (2008). Using the power of hope to cope with dying. The four stages of hope. Sanger, CA: Quill Driver Books.

Feeley, N., \& Gottlieb, L.N. (2000). Nursing approaches for working with family strengths and resources. Journal of Family Nursing, 6(1), 9-24.

Feeley, N., \& Gottlieb, L.N. (1998). Classification systems for health concerns, nursing strategies and client outcomes: Nursing practice with families who have a child with a chronic illness. Canadian Journal of Nursing Research, 30, 45-59.

Fitzpatrick, J.J. (2005). Nursing knowledge development: Relationship to science and professional practice. In J.J. Fitzpatrick, \& A.L. Whall (Eds.), Conceptual models of nursing. Analysis and application (4th ed., pp.1-4). Upper Saddle River, NJ: Pearson Education Inc.

Gallant, M.H., Beaulieu, M.C., \& Carnevale, F.A. (2002). Partnership: An analysis of the concept within the nurse-client relationship. Journal of Advanced Nursing, 40(2), 149-157.

Gottlieb, L.N. (1997). Health promoters: Two contrasting styles in community nursing. In L.N. Gottlieb, \& H. Ezer (Eds.), $A$ perspective on health, family, learning, and collaborative nursing. A collection of writings on the McGill model of nursing. (pp.87100). Montreal, QC: McGill University School of Nursing.

Gottlieb, L.N. (1995). A blueprint for the development of the profession of nursing: The legacy of F. Moyra Allen and Joan Gilchrist. Canadian Journal of Nursing Research, 27(3), 5-7.

Gottlieb, L.N., \& Feeley, N., with Dalton, C. (2005). The collaborative partnership approach to care. A delicate balance. Toronto, ON: Elsevier Canada.

Gottlieb, L.N., \& Gottlieb, B. (2007). The developmental/health framework within the McGill model of nursing: "Laws of nature" guiding whole person care. Advances in Nursing Science, 30(1), E43-E57.

Gottlieb, L., \& Rowat, K. (1987). The McGill model of nursing: A practice-derived model. Advances in Nursing Science, 9(4), 5161.

Gros, C., \& Ezer, H. (1997). Promoting inquiry and nurse-client collaboration: A unique approach to teaching and learning. In L.N. Gottlieb, \& H. Ezer (Eds.), A perspective on health, family, learning, and collaborative nursing. A collection of writings on the McGill model of nursing. (pp.219-225). Montreal, QC: McGill University School of Nursing.

Kessler, D. (2007). The needs of the dying. A guide for bringing hope, comfort, and love to life's final chapter. (10th Anniversary ed.). New-York, NY: Harper Collins Publishers.

Kristjanson, L.J., \& Aoun, S. (2004). Palliative care for families: Remembering the hidden patients. Canadian Journal of Psychiatry, 49, 359-365.

Leahey, M., \& Harper-Jaques, S. (1996). Family-nurse relationships: core assumptions and clinical implications. Journal of Family Nursing, 2(2), 133-151.

Lowey, S. (2008). Letting go before death: A concept analysis. Journal of Advanced Nursing, 63, 208-215.

Meiers, S.J., \& Brauer, D.J. (2008). Existential caring in the family health experience: A proposed conceptualization. Scandinavian Journal of Caring Sciences, 22, 110-117.

Meleis, A.I. (2007). Theoretical nursing. Development and progress. (4th ed.). Philadelphia, PA: Lippincott Williams \& Wilkins.
Parse, R.R. (1990). Health: A personal commitment. Nursing Science Quarterly, 3(3), 136-140.

Parse, R.R. (1998). The human becoming school of thought. A perspective for nurses and other health professionals. Thousand Oaks, CA: Sage Publications.

Pastrana, T., Junger, S., Ostgathe, C., Elsner, F., \& Radbruch, L. (2008). A matter of definition-key elements identified in a discourse analysis of definitions of palliative care. Palliative Medicine, 22, 222-232.

Perry, B. (1998). Moments in time: Images of exemplary nursing care. Ottawa, ON: Canadian Nurses Association.

Pugnaire, C. (1981). Nursing: the Science of Health-Promoting Interaction. Unpublished Master's study School of Nursing, McGill University, Montreal, Canada.

Pugnaire-Gros, C., \& Young, L.E. (2007). Teaching the McGill model of nursing and client-centred care: Collaborative strategies for staff education and development. In L.E. Young \& B.L. Patterson (Eds.), Teaching nursing: Developing a student-centred learning environment (pp.189-220). Philadelphia: Lippincott Williams \& Wilkins.

Quill, T.E., Arnold, R., \& Back, A.L. (2009). Discussing treatement preferences with patients who want "everything". Annals of Internal Medicine, 151, 345-349.

Saunders, C. (2000). The evolution of palliative care. Patient Education and Counseling, 41, 7-13.

Sjolander, C., Hedberg, B., \& Ahlstrom, G. (2011). Striving to be prepared for the painful: Management strategies following a family members' diagnosis of advanced cancer. BMC Nursing, 10(18), 1-8.

Société canadienne du cancer, Comité directeur sur les statistiques sur le cancer. (2011). Statistiques canadiennes sur le cancer 2011. Toronto, ON: Société canadienne du cancer. Récupéré à http:// www.cancer.ca/Canada-wide/About\%20cancer/Cancer\%20 statistics.aspx?sc_lang=fr-ca

Stuart, M. (2002). F. Moyra Allen: A life in nursing, 1921-1996. Journal of Family Nursing, 8(2), 157-165.

Thompson, G.N., McClement, S.E., \& Daeninck, P.J. (2006). "Changing lanes": Facilitating the transition from curative to palliative care. Journal of Palliative Care, 22(2), 91-98.

Thorne, S. (2007). Conceptualizing the purpose of nursing: philosophical challenges in creating meaningful theoretical learning experiences. In L.E. Young \& B.L. Patterson (Eds.), Teaching nursing: Developing a student-centered learning environment (pp.347-363). Philadelphia: Lippincott Williams \& Wilkins.

Twycross, R. (2007). Patient care: Past, present, and future. Omega: The Journal of Death and Dying, 56(1), 7-19.

Willis, D.G., Grace, P.J., \& Roy, C. (2008). A central unifying focus for the discipline: Facilitating humanization, meaning, choice, quality of life, and healing in living and dying. Advances in Nursing Science, 31(1), E28-E40.

Wilson, D.M., Truman, C.D., Thomas, R., Fainsinger, R., KovacsBurns, K., Froggatt, K., et al. (2009). The rapidly changing location of death in Canada, 1994-2004. Social Science \& Medicine, 68, 1752-1758.

Wright, D., Brajtman, S., \& Bitzas, V. (2009). Human relationships at the end of life: An ethical ontology for practice. Journal of Hospice and Palliative Nursing, 11(4), 219-227.

Wright, L.M., \& Leahey, M. (2005). Nurses and families. A guide to family assessment and intervention. (4th ed.). Philadelphia, PA: F.A. Davis Company. 